PROCEEDINGS OF THE

AMERICAN MATHEMATICAL SOCIETY

Volume 133, Number 9, Pages 2569-2575

S 0002-9939(05)07978-5

Article electronically published on March 31, 2005

\title{
UNIFORMLY BOUNDED LIMIT OF FRACTIONAL HOMOMORPHISMS
}

\author{
PEDRO J. MIANA
}

(Communicated by Jonathan M. Borwein)

\begin{abstract}
We show that a bounded homomorphism $T: L_{\omega}^{1}\left(\mathbb{R}^{+}\right) \rightarrow \mathcal{A}$ is equivalent to a uniformly bounded family of fractional homomorphisms $T_{\alpha}$ : $A C_{\omega}^{(\alpha)}\left(\mathbb{R}^{+}\right) \rightarrow \mathcal{A}$ for any $\alpha>0$. We add this characterization to the WidderArendt-Kisyński theorem and relate it to $\alpha$-times integrated semigroups.
\end{abstract}

\section{INTRODUCTION}

Let $\mathcal{A}$ be a Banach algebra (with or without identity). For $\Omega \subset \mathbb{C}$, a family $\left(r_{\lambda}\right)_{\lambda \in \Omega}$ of elements of $\mathcal{A}$ is called a pseudo-resolvent if the equation $r_{\lambda}-r_{\mu}=$ $(\mu-\lambda) r_{\lambda} r_{\mu}$ holds for $\lambda, \mu \in \Omega$. An example of a pseudo-resolvent is the following. Let $\mathbb{R}, \mathbb{R}^{+}$and $\mathbb{C}$ be the sets of real, positive real and complex numbers, respectively. For each $\lambda \in \mathbb{R}$, denote by $\epsilon_{\lambda}$ the function

$$
\epsilon_{\lambda}(t)=e^{\lambda t}, \quad t \in \mathbb{R}^{+} .
$$

Take $\omega \in \mathbb{R}^{+} \cup\{0\}$ and let $L_{\omega}^{1}\left(\mathbb{R}^{+}\right)$be the usual Banach algebra with norm given by

$$
\|f\|_{w}:=\int_{0}^{\infty}|f(t)| e^{\omega t} d t<+\infty
$$

and the convolution $f * g(t):=\int_{0}^{t} f(t-s) g(s) d s$, with $t \geq 0$, as its product. Then $\left(\epsilon_{-\lambda}\right)_{\lambda \in(\omega,+\infty)}$ is a pseudo-resolvent in $L_{\omega}^{1}\left(\mathbb{R}^{+}\right)$and verifies

$$
\|\underbrace{\epsilon_{-\lambda} * \cdots * \epsilon_{-\lambda}}_{n \text { times }}\|_{\omega}=\frac{1}{(\lambda-\omega)^{n}}, \quad \lambda \in(\omega,+\infty), n \in \mathbb{N} .
$$

Moreover, the set $\left(\epsilon_{-\lambda}\right)_{\lambda \in(\omega,+\infty)}$ is linearly dense in $L_{\omega}^{1}\left(\mathbb{R}^{+}\right)$.

The next result shows the equivalence between a homomorphism $T: L_{\omega}^{1}\left(\mathbb{R}^{+}\right)$ $\rightarrow \mathcal{A}$ and a class of pseudo-resolvents; in other words, the family $\left(\epsilon_{-\lambda}\right)_{\lambda \in(\omega,+\infty)}$ is universal for this class of pseudo-resolvents. We present here an early version; see 8] and [3] which is included in [9, Theorem 5.1]. Recently, this result has been called the Widder-Arendt-Kisyński theorem; see for example [5, Theorem 1.1].

Received by the editors February 1, 2003.

2000 Mathematics Subject Classification. Primary 47D62; Secondary 26A33, 46J25.

Key words and phrases. Pseudo-resolvents, homomorphisms, integrated semigroups.

This work was supported by a grant from Programa Europa, CAI, 2002. This paper was made during a visit to the Charles University in Prague. The author thanks Dr. Eva Fasangova and the Analysis Mathematical Department for the stay in Prague.

(C)2005 American Mathematical Society Reverts to public domain 28 years from publication 
Theorem 1.1 ([8]). Let $\mathcal{A}$ be a Banach algebra, let $\omega \geq 0$, let $\left(r_{\lambda}\right)_{\lambda \in(\omega,+\infty)}$ be a pseudo-resolvent in $\mathcal{A}$ and let

$$
M=\sup \left\{(\lambda-\omega)^{n}\left\|r_{\lambda}^{n}\right\|: n \in \mathbb{N}, \lambda \in(\omega,+\infty)\right\} .
$$

Then the following conditions are equivalent:

(i) $M<+\infty$.

(ii) There exists a continuous homomorphism $T: L_{\omega}^{1}\left(\mathbb{R}^{+}\right) \rightarrow \mathcal{A}$ such that $T\left(\epsilon_{-\lambda}\right)=r_{\lambda}$ for each $\lambda \in(\omega,+\infty)$.

Furthermore, if a continuous homomorphism $T: L_{\omega}^{1}\left(\mathbb{R}^{+}\right) \rightarrow \mathcal{A}$ satisfying $T\left(\epsilon_{-\lambda}\right)$ $=r_{\lambda}$ for each $\lambda \in(\omega,+\infty)$ exists, then it is unique and $\|T\|=M$.

This theorem has many interesting applications, as W. Chojnacki pointed out, and it is equivalent to Hille-Yosida theorem; see [3] and [4. Another different proof of this result is given in [2, where the author uses Yosida approximation in a more general setting. A generalization of [9] Theorem 5.1] is presented in 5] improving Kisyński's arguments.

In this paper, we arrive at Theorem 1.1 with an original point of wiev. We show that a bounded homomorphism $T: L_{\omega}^{1}\left(\mathbb{R}^{+}\right) \rightarrow \mathcal{A}$ is equivalent to a uniformly bounded family of homomorphisms $T_{\alpha}: A C_{\omega}^{(\alpha)}\left(\mathbb{R}^{+}\right) \rightarrow \mathcal{A}$ for any $\alpha>0$; see Theorem 3.1. Fractional Banach algebras $A C_{\omega}^{(\alpha)}\left(\mathbb{R}^{+}\right)$are included in $L_{\omega}^{1}\left(\mathbb{R}^{+}\right)$and defined in the second section. The proof of this main result is based on Theorem 1.1 .

A family of fractional algebras similar to $A C_{\omega}^{(\alpha)}\left(\mathbb{R}^{+}\right)$was introduced in [10], working with $\alpha$-times integrated semigroups. Integrated semigroups are considered in the fourth section to complete a different approach to the Widder-ArendtKisyński theoreom in which Lipschitz and Hölder continuous functions appear; see [1, Theorem 1.1], [6] Theorem 2.6], [9 Corollary 7.2], or 7, Theorem 3.2]. Lipschitz functions are considered in the integrated version of the Widder's characterization of Laplace transforms on arbitrary Banach spaces.

Actually, $\alpha$-times integrated semigroups (in the case $\alpha=1$ ) were also used to prove Theorem 1.1 with $\omega=0$ in [8 Theorem 4.2]. However, we present here a different technique since we consider $\alpha$-times integrated semigroups for any $\alpha>0$ and the limit when $\alpha$ goes to zero.

\section{Fractional Banach algebras}

Suppose $f \in \mathcal{D}_{+}$, where $C_{c}^{\infty}[0, \infty) \equiv \mathcal{D}_{+}$is the set of infinitely differentiable functions with compact support on $[0, \infty)$. The Weyl fractional integral of $f$ of order $\alpha>0, W^{-\alpha} f$, is defined by

$$
W^{-\alpha} f(u)=\frac{1}{\Gamma(\alpha)} \int_{u}^{\infty}(t-u)^{\alpha-1} f(t) d t, \quad u \geq 0,
$$

with $\alpha>0$; see for example [11]. This operator $W^{-\alpha}: \mathcal{D}_{+} \rightarrow \mathcal{D}_{+}$is one-to-one and onto. Its inverse, $W^{\alpha}$, is called a Weyl fractional derivate (of $f$ ) of order $\alpha$ and $W^{\alpha}=(-1)^{n} \frac{d^{n}}{d t^{n}} W^{-(n-\alpha)}$ with $n \in \mathbb{N}$ and $n>\alpha$.

Theorem 2.1. Suppose $\alpha>0$ and $\omega \geq 0$. If $f \in \mathcal{D}_{+}$, then the formula

$$
\|f\|_{(\alpha, \omega)}:=\frac{1}{\Gamma(\alpha+1)} \int_{0}^{\infty} t^{\alpha} e^{\omega t}\left|W^{\alpha} f(t)\right| d t
$$


defines a norm in $\mathcal{D}_{+}$such that

$$
\|f * g\|_{(\alpha, \omega)} \leq\left(2^{\alpha+1}-1\right)\|f\|_{(\alpha, \omega)}\|g\|_{(\alpha, \omega)},
$$

with $f, g \in \mathcal{D}_{+}$. We denote by $A C_{\omega}^{(\alpha)}\left(\mathbb{R}^{+}\right)$the Banach algebra obtained by the completion of $\mathcal{D}_{+}$in this norm.

Proof. The proof is similar to [10, Theorem 1.2].

Remark 2.2. For $\alpha=0$, it is clear that $A C_{\omega}^{(0)}\left(\mathbb{R}^{+}\right)=L_{\omega}^{1}\left(\mathbb{R}^{+}\right)$and $A C_{\omega}^{(\beta)}\left(\mathbb{R}^{+}\right) \hookrightarrow$ $A C_{\omega}^{(\alpha)}\left(\mathbb{R}^{+}\right)$holds with $0 \leq \alpha \leq \beta$.

It is easy to check that $W^{\alpha}\left(\epsilon_{-\lambda}\right)=\lambda^{\alpha} \epsilon_{-\lambda}$ with $\alpha \in \mathbb{R}$ and $\lambda>0$. Functions $\left(\epsilon_{-\lambda}\right)_{\lambda \in(\omega,+\infty)}$ belong to $A C_{\omega}^{(\alpha)}\left(\mathbb{R}^{+}\right)$with $\alpha \geq 0$ and

$$
\left\|\epsilon_{-\lambda}\right\|_{(\alpha, \omega)}=\frac{\lambda^{\alpha}}{(\lambda-\omega)^{\alpha+1}}, \quad \lambda \in(\omega,+\infty) .
$$

Note that $\left\|\epsilon_{-\lambda}\right\|_{(\alpha, \omega)} \rightarrow\left\|\epsilon_{-\lambda}\right\|_{\omega}$ when $\alpha \rightarrow 0^{+}$and $\lambda \in(\omega,+\infty)$. Moreover, $\left(\epsilon_{-\lambda}\right)_{\lambda \in(\omega, \infty)}$ is a pseudo-resolvent in $A C_{\omega}^{(\alpha)}\left(\mathbb{R}^{+}\right)$and $\left(n \epsilon_{-n}\right)_{n>\omega}$ is a bounded approximate identity in $A C_{\omega}^{(\alpha)}\left(\mathbb{R}^{+}\right)$for any $\alpha \geq 0$.

Proposition 2.3. The set $\left(\epsilon_{-\lambda}\right)_{\lambda \in(\omega,+\infty)}$ is linearly dense in $A C_{\omega}^{(\alpha)}\left(\mathbb{R}^{+}\right)$with $\alpha, \omega \geq 0$.

Proof. It is a consequence of $W^{\alpha}\left(\epsilon_{-\lambda}\right)=\lambda^{\alpha} \epsilon_{-\lambda}$ with $\alpha \geq 0, \lambda>\omega$ and [5, Proposition 2.2].

\section{MAin RESUlt}

Theorem 3.1. Let $\mathcal{A}$ be a Banach algebra.

(i) If there exists a continuous homomorphism $T: L_{\omega}^{1}\left(\mathbb{R}^{+}\right) \rightarrow \mathcal{A}$, then there exists a family of continuous homomorphisms $T_{\alpha}: A C_{\omega}^{(\alpha)}\left(\mathbb{R}^{+}\right) \rightarrow \mathcal{A}$ for each $\alpha>0$ such that $T_{\alpha}\left(\epsilon_{-\lambda}\right)=T\left(\epsilon_{-\lambda}\right)$ for each $\lambda \in(\omega,+\infty)$ and $\left\|T_{\alpha}\right\| \leq\|T\|$ for any $\alpha>0$.

(ii) Conversely, if for each $\alpha>0$ there exist a countinuous homomorphisms $T_{\alpha}: A C_{\omega}^{(\alpha)}\left(\mathbb{R}^{+}\right) \rightarrow \mathcal{A}$ such that $T_{\alpha}\left(\epsilon_{-\lambda}\right)$ does not depend on $\alpha$ for each $\lambda \in(\omega,+\infty)$ and $\lim \sup _{\alpha \rightarrow 0^{+}}\left\|T_{\alpha}\right\|<+\infty$, then there exists a unique continuous homomorphism $T: L_{\omega}^{1}\left(\mathbb{R}^{+}\right) \rightarrow \mathcal{A}$ such that $T\left(\epsilon_{-\lambda}\right)=T_{\alpha}\left(\epsilon_{-\lambda}\right)$ for each $\lambda \in(\omega,+\infty)$ and $\|T\| \leq$ $\lim \sup _{\alpha \rightarrow 0^{+}}\left\|T_{\alpha}\right\|$.

Proof. The first part follows from Remark 2.2. To prove (ii), we use Theorem 1.1. We define $r_{\lambda}:=T_{\alpha}\left(\epsilon_{\lambda}\right)$ for each $\lambda>\omega$. The family $\left(r_{\lambda}\right)_{\lambda \in(\omega, \infty)}$ is well defined and is a pseudo-resolvent in $\mathcal{A}$. For $n \in \mathbb{N}$ and $\lambda \in(\omega,+\infty)$,

$$
\left\|r_{\lambda}^{n}\right\| \leq\left\|T_{\alpha}\right\|\|\underbrace{\epsilon_{\lambda} * \cdots * \epsilon_{\lambda}}_{n \text { times }}\|_{(\alpha, \omega)}
$$

We apply Theorem 2.1 and get that

$$
\|\underbrace{\epsilon_{\lambda} * \cdots * \epsilon_{\lambda}}_{n \text { times }}\|_{(\alpha, \omega)} \leq\left(2^{\alpha+1}-1\right)^{n-1}\left\|\epsilon_{\lambda}\right\|_{(\alpha, \omega)}^{n}=\left(2^{\alpha+1}-1\right)^{n-1} \frac{\lambda^{n \alpha}}{(\lambda-\omega)^{n(\alpha+1)}},
$$

where we use (1). Now we obtain

$$
\left\|r_{\lambda}^{n}\right\| \leq \lim _{\alpha \rightarrow 0}\left(\left(2^{\alpha+1}-1\right)^{n-1} \frac{\lambda^{n \alpha}}{(\lambda-\omega)^{n(\alpha+1)}}\right) \limsup _{\alpha \rightarrow 0^{+}}\left\|T_{\alpha}\right\|,
$$


and we get

$$
\sup \left\{(\lambda-\omega)^{n}\left\|r_{\lambda}^{n}\right\|: n \in \mathbb{N}, \lambda \in(\omega,+\infty)\right\} \leq \limsup _{\alpha \rightarrow 0^{+}}\left\|T_{\alpha}\right\|<+\infty .
$$

By Theorem 1.1, there exists a unique continuous homomorphism $T: L_{\omega}^{1}\left(\mathbb{R}^{+}\right) \rightarrow \mathcal{A}$ such that $T\left(\epsilon_{-\lambda}\right)=r_{\lambda}=T_{\alpha}\left(\epsilon_{-\lambda}\right)$ for each $\lambda \in(\omega,+\infty)$ and $\|T\| \leq \lim \sup _{\alpha \rightarrow 0^{+}}\left\|T_{\alpha}\right\|$.

Remark 3.2. Since the set $\left(\epsilon_{-\lambda}\right)_{\lambda \in(\omega,+\infty)}$ is linearly dense in $A C_{\omega}^{(\alpha)}\left(\mathbb{R}^{+}\right)$(Proposition 2.3), $T_{\alpha}(f)=T(f)$ for any $f \in A C_{\omega}^{(\alpha)}\left(\mathbb{R}^{+}\right)$and $\alpha>0$. Also, we may combine (i) and (ii) to get

$$
\|T\|=\limsup _{\alpha \rightarrow 0^{+}}\left\|T_{\alpha}\right\|=\sup _{\alpha>0}\left\|T_{\alpha}\right\|,
$$

where we use $\left\|T_{\beta}\right\| \leq\left\|T_{\alpha}\right\|$ with $\beta \geq \alpha>0$.

\section{Connection with integrated Semigroups}

Widder's characterization of Laplace transforms of real-valued bounded functions which states that for $r \in C^{(\infty)}(0, \infty)$ there exists $f \in L^{\infty}(0, \infty)$ such that

$$
r(\lambda)=\int_{0}^{\infty} e^{-\lambda t} f(t) d t, \quad \lambda>0,
$$

if and only if

$$
\sup \left\{\lambda^{n+1} \frac{\left|r^{(n)}(\lambda)\right|}{n !}: \lambda>0, n \in \mathbb{N}\right\}<+\infty,
$$

(see [12]) was considered by W. Arendt on arbitrary Banach space $X$. An integrated version of this result is given in terms of a function $F:[0, \infty) \rightarrow X$ such that $F(0)=0,\|F(t+h)-F(t)\| \leq M h,(t, h \geq 0)$ and

$$
r(\lambda)=\lambda \int_{0}^{\infty} e^{-\lambda t} F(t) d t, \quad \lambda>0
$$

see [1. Theorem 1.1]. This result was improved by M. Hieber who showed that for any $\alpha \in(0,1]$ there exists a function $F_{\alpha}:[0, \infty) \rightarrow X$ such that $F_{\alpha}(0)=0$, $\left\|F_{\alpha}(t+h)-F_{\alpha}(t)\right\| \leq M h^{\alpha},(t, h \geq 0)$ and

$$
r(\lambda)=\lambda^{\alpha} \int_{0}^{\infty} e^{-\lambda t} F(t) d t, \quad \lambda>0
$$

see 7, Theorem 3.2]. If $r$ is a pseudo-resolvent in a Banach algebra $\mathcal{A}$, then the function $F_{\alpha}$ is an $\alpha$-times integrated semigroup in $\mathcal{A}$. We now give here the definition of $\alpha$-times integrated semigroups in a Banach algebra $\mathcal{A}$, since they are usually considered in the setting of linear and bounded operators on a Banach space $X$; see for example [7].

Definition 4.1. For any $\alpha>0$, an $\alpha$-times integrated semigroup $s_{\alpha}(\cdot):[0, \infty) \rightarrow \mathcal{A}$ is a continuous mapping with $s_{\alpha}(0)=0$ and

(4.1) $s_{\alpha}(t) s_{\alpha}(s)=\frac{1}{\Gamma(\alpha)}\left(\int_{t}^{t+s}(t+s-r)^{\alpha-1} s_{\alpha}(r) d r-\int_{0}^{s}(t+s-r)^{\alpha-1} s_{\alpha}(r) d r\right)$,

with $t, s \geq 0$. 
If $\left\|s_{\alpha}(t)\right\| \leq C e^{\omega t}$ for $C, t \geq 0$ and $\omega \in \mathbb{R}$, then condition (2) is equivalent (by Laplace transform) to $r_{\lambda}:=\lambda^{\alpha} \int_{0}^{\infty} e^{-\lambda t} s_{\alpha}(t) d t$, with $\lambda>\omega$ a pseudo-resolvent in $\mathcal{A}$; see the analogous proof in Banach space in [7]. If $\left(s_{\alpha}(t)\right)_{t \geq 0}$ is an $\alpha$-times integrated semigroup in $\mathcal{A}$, then $\left(s_{\nu}(t)\right)_{t \geq 0}$ with

$$
s_{\nu}(t):=\frac{1}{\Gamma(\nu-\alpha)} \int_{0}^{t}(t-s)^{\nu-\alpha-1} s_{\alpha}(s) d s, \quad t \geq 0
$$

is a $\nu$-times integrated semigroup with $\nu>\alpha$ in $\mathcal{A}$. The set of Bochner-Riesz functions $\left(R_{t}^{\nu}\right)_{t \geq 0}$, i.e,

$$
R_{t}^{\nu}(s):=\frac{(t-s)^{\nu-1}}{\Gamma(\nu)} \chi_{(0, t)}(s), \quad t \geq 0
$$

is an example of a $\nu$-times integrated semigroup in $A C_{\omega}^{(\alpha)}\left(\mathbb{R}^{+}\right)$for $\nu>\alpha, \omega \geq 0$ and

$$
\left\|R_{t}^{\nu}\right\|_{(\alpha, \omega)} \leq \frac{t^{\nu} e^{t \omega}}{\Gamma(\nu+1)}, \quad t \geq 0
$$

Now, we may add in the Widder-Arendt-Kisyński theoreom more conditions related to fractional homomorphisms and $\alpha$-times integrated semigroups.

Theorem 4.2. Let $\mathcal{A}$ be a Banach algebra, let $\omega$ be a non-negative number, let $\left(r_{\lambda}\right)_{\lambda \in(\omega,+\infty)}$ be a pseudo-resolvent in $\mathcal{A}$ and let

$$
M=\sup \left\{(\lambda-\omega)^{n}\left\|r_{\lambda}^{n}\right\|: n \in \mathbb{N}, \lambda \in(\omega,+\infty)\right\} .
$$

Then the following conditions are equivalent:

(i) $M<+\infty$.

(ii) There exists a continuous homomorphism $T: L_{\omega}^{1}\left(\mathbb{R}^{+}\right) \rightarrow \mathcal{A}$ such that $T\left(\epsilon_{-\lambda}\right)=r_{\lambda}$ for each $\lambda \in(\omega,+\infty)$.

(iii) For any $\alpha>0$, there exists an $\alpha$-times integrated semigroup $\left(s_{\alpha}(t)\right)_{t \geq 0} \subset \mathcal{A}$ such that $\left\|s_{\alpha}(t)\right\| \leq \frac{C}{\Gamma(\alpha+1)} t^{\alpha} e^{\omega t}$ with $C, t \geq 0$ and $r_{\lambda}=\lambda^{\alpha} \int_{0}^{\infty} e^{-\lambda t} s_{\alpha}(t) d t$, with $\lambda>\omega$.

(iv) For any $\alpha>0$, there exists a continuous homomorphism $T_{\alpha}: A C_{\omega}^{(\alpha)}\left(\mathbb{R}^{+}\right) \rightarrow$ $\mathcal{A}$ such that $T_{\alpha}\left(\epsilon_{-\lambda}\right)=r_{\lambda}$ for each $\lambda \in(\omega,+\infty)$ and $\sup _{\alpha>0}\left\|T_{\alpha}\right\|<+\infty$.

Furthermore, if a continuous homomorphism $T: L_{\omega}^{1}\left(\mathbb{R}^{+}\right) \rightarrow \mathcal{A}$ such that $T\left(\epsilon_{-\lambda}\right)$ $=r_{\lambda}$ for each $\lambda \in(\omega,+\infty)$ exists, then it is unique, $T(f)=T_{\alpha}(f)$ for $f \in$ $A C_{\omega}^{(\alpha)}\left(\mathbb{R}^{+}\right)$and

$$
M=\|T\|=\sup _{\alpha>0}\left\|T_{\alpha}\right\|=\inf \left\{C:\left\|s_{\alpha}(t)\right\| \leq C \frac{t^{\alpha} e^{\omega t}}{\Gamma(\alpha+1)}, t \geq 0\right\} .
$$

Proof. (ii) $\Rightarrow$ (iii) Take $\left(R_{t}^{\alpha}\right)_{t>0}$ to be the family of Bochner-Riesz functions of order $\alpha>0$. Then $\left(R_{t}^{\alpha}\right)_{t \geq 0} \subset L_{\omega}^{1}\left(\mathbb{R}^{+}\right)$and we define $s_{\alpha}(t):=T\left(R_{t}^{\alpha}\right)$, for $t>0$ and $s_{\alpha}(0):=0$. Since $\left(R_{t}^{\alpha}\right)_{t>0}$ is an $\alpha$-times integrated semigroup in $L_{\omega}^{1}\left(\mathbb{R}^{+}\right),\left(s_{\alpha}(t)\right)_{t \geq 0}$ is an $\alpha$-times integrated semigroup in $\mathcal{A}$ and

$$
\left\|s_{\alpha}(t)\right\| \leq\|T\|\left\|R_{t}^{\alpha}\right\|_{\omega} \leq\|T\| \frac{t^{\alpha}}{\Gamma(\alpha+1)} e^{\omega t} .
$$

By continuity of $T$, we have for $\lambda>\omega$,

$$
\lambda^{\alpha} \int_{0}^{\infty} e^{-\lambda t} s_{\alpha}(t) d t=\lambda^{\alpha} T\left(\int_{0}^{\infty} e^{-\lambda t} R_{t}^{\alpha} d t\right)=\lambda^{\alpha} T\left(W^{-\alpha} \epsilon_{\lambda}\right)=T\left(\epsilon_{\lambda}\right)=r_{\lambda} .
$$


(iii) $\Rightarrow$ (iv) We define $T_{\alpha}: A C_{\omega}^{(\alpha)}\left(\mathbb{R}^{+}\right) \rightarrow \mathcal{A}$ by

$$
T_{\alpha}(f):=\int_{0}^{\infty} W^{\alpha} f(t) s_{\alpha}(t) d t, \quad f \in \mathcal{D}_{+} .
$$

Following the same arguments as in [10, Theorem 3.1], we prove that $T_{\alpha}$ are continuous homomorphisms, $\left\|T_{\alpha}\right\| \leq C$ for any $\alpha>0$ and $\sup _{\alpha>0}\left\|T_{\alpha}\right\| \leq C<+\infty$. Since $\epsilon_{\lambda} \in A C_{\omega}^{(\alpha)}\left(\mathbb{R}^{+}\right)$for $\lambda>\omega$ and $W^{\alpha}\left(\epsilon_{\lambda}\right)=\lambda^{\alpha} \epsilon_{\lambda}$, we have $T_{\alpha}\left(\epsilon_{\lambda}\right)=r_{\lambda}$ for $\lambda>\omega$.

(iv) $\Rightarrow$ (ii) It is proved by Theorem 3.1(ii).

If a continuous homomorphism $T: L_{\omega}^{1}\left(\mathbb{R}^{+}\right) \rightarrow \mathcal{A}$ satisfying $T\left(\epsilon_{-\lambda}\right)=r_{\lambda}$ for each $\lambda \in(\omega,+\infty)$ exists, then $A C_{\omega}^{(\alpha)}\left(\mathbb{R}^{+}\right) \hookrightarrow L_{\omega}^{1}\left(\mathbb{R}^{+}\right)$and $T(f)=T_{\alpha}(f)$ for $f \in A C_{\omega}^{(\alpha)}\left(\mathbb{R}^{+}\right)$. Moreover, if we collect in the proof the following inequalities,

$$
\|T\|=M=\sup _{\alpha>0}\left\|T_{\alpha}\right\| \leq \inf \left\{C:\left\|s_{\alpha}(t)\right\| \leq C \frac{t^{\alpha} e^{\omega t}}{\Gamma(\alpha+1)}, t \geq 0\right\} \leq\|T\|,
$$

we finally get the equality.

Remark 4.3. If $0<\alpha \leq 1$, we use $s_{\alpha}(t)=T\left(R_{t}^{\alpha}\right)(t>0)$ to obtain

$$
\left\|s_{\alpha}(t+h)-s_{\alpha}(t)\right\| \leq 2\|T\| \frac{h^{\alpha}}{\Gamma(\alpha+1)} e^{\omega(t+h)},
$$

with $t, h>0$; see also [7, Corollary 3.3].

\section{ACKNOWLEDGMENT}

I thank the referee for careful reading of this paper and valuable suggestions and references that lead to its improvement.

\section{REFERENCES}

1. W. Arendt, Vector-Valued Laplace Transforms and Cauchy Problems, Israel J. Math. 59(3) (1987), 327-352. MR0920499 (89a:47064)

2. A. Bobrowski, On the Yosida Approximation and the Widder-Arendt Representation Theorem, Studia Math. 124 (1997), 281-290. MR.1456426 (99b:47058)

3. W. Chojnacki, On the Equivalence of a Theorem of Kisyński and the Hille-Yosida Generation Theorem, Studia Math. 126(2) (1998), 491-497. MF1415577 (98d:46050)

4. W. Chojnacki, Multiplier Algebras, Banach Bundles, and One-Parameter Semigroups, Ann. Scuola. Norm. Sup. Pisa Cl. Sci. (4) 28 (1999), 287-322. MR.1736230 (2002a:46066)

5. W. Chojnacki, A Generalization of the Widder-Arendt Theorem, Proc. Edinburgh. Math. Soc. 45 (2002), 161-179. MR 1884610 (2002k:44002)

6. B. Hennig and F. Neubrander, On Representations, Inversions, and Approximations of Laplace Transforms in Banach Spaces, Appl. Anal. 49 (1993), 151-170. MR1289741 (95m:46053)

7. M. Hieber, Laplace Transforms and $\alpha$-Times Integrated Semigroups, Forum Math 3 (1991), 595-612. MF1130001 (92k:47075)

8. J. Kisyński, The Widder Spaces, Representations of the Convolution Algebra $L^{1}\left(\mathbb{R}^{+}\right)$and one Parameter Semigroups of Operators, Preprint no. 558, Institute of Mathematics, Polish Academy of Sciences, Warsaw, June 1998.

9. J. Kisyński, Around Widder's Characterization of the Laplace Transform of an Element of $L^{\infty}\left(\mathbb{R}^{+}\right)$, Ann. Polon. Math. 74 (2000), 161-200. MF $1808994(2002 \mathrm{j}: 44001)$

10. P.J. Miana, $\alpha$-Times Integrated Semigroups and Fractional Derivation, Forum Math. 14 (2002), 23-46. MR1880193 (2003k:47059) 
11. K.S. Miller and B. Ross, An Introduction to the Fractional Calculus and Fractional Differential Equations, Wiley, New York, 1993. MR1219954 (94e:26013)

12. D. V. Widder, The Laplace Transform, Princeton Univ. Press, 1946. MR0005923 (3:232d)

Departamento de Matemáticas, Facultad de Ciencias, Universidad de Zaragoza, 50009 Zaragoza, SPAIN

E-mail address: pjmiana@unizar.es 